\title{
Slit Proteins Regulate Distinct Aspects of Retinal Ganglion Cell Axon Guidance within Dorsal and Ventral Retina
}

\author{
Hannah Thompson, ${ }^{\star}$ Olivier Camand, ${ }^{\star}$ David Barker, and Lynda Erskine \\ Divisions of Visual Science and Molecular Genetics, Institute of Ophthalmology, University College London, London EC1V 9EL, United Kingdom
}

An early step in the formation of the optic pathway is the directed extension of retinal ganglion cell (RGC) axons into the optic fiber layer (OFL) of the retina in which they project toward the optic disc. Using analysis of knock-out mice and in vitro assays, we found that, in the mammalian retina, Slit1 and Slit2, known chemorepellents for RGC axons, regulate distinct aspects of intraretinal pathfinding in different regions of the retina. In ventral and, to a much lesser extent, dorsal retina, Slits help restrict RGC axons to the OFL. Additionally, within dorsal retina exclusively, Slit2 also regulates the initial polarity of outgrowth from recently differentiated RGCs located in the retinal periphery. This regional specificity occurs despite the fact that Slits are expressed throughout the retina, and both dorsal and ventral RGCs are responsive to Slits. The gross morphology and layering of the retina of the slit-deficient retinas is normal, demonstrating that these distinct guidance defects are not the result of changes in the organization of the tissue. Although displaced or disorganized, the aberrant axons within both dorsal and ventral retina exit the eye. We also have found that the lens, which because of its peripheral location within the developing eye is ideally located to influence the initial direction of RGC axon outgrowth, secretes Slit2, suggesting this is the source of Slit regulating OFL development. These data demonstrate clearly that multiple mechanisms exist in the retina for axon guidance of which Slits are an important component.

Key words: Robo; Slit; growth cone; axon guidance; visual system; retinal ganglion cell

\section{Introduction}

After differentiation, retinal ganglion cells (RGCs) extend their axons in a highly directed, radial manner toward the optic disc. As they extend through the retina, the axons are restricted to the optic fiber layer (OFL) at the inner surface of the retina by the balance between the growth-promoting properties of the radial glial end feet localized to this region and inhibitory cues present within the outer retina (Stier and Schlosshauer, 1995, 1998).

The molecular mechanisms regulating intraretinal pathfinding are beginning to be unraveled. In rodents, a centralperipheral wave of chondroitin sulfate proteoglycan (CSPG) expression controls the initial polarity of outgrowth from recently differentiated RGCs, directing the axons away from the retinal periphery (Brittis et al., 1992; Brittis and Silver, 1995). More centrally, EphB2/B3, BMP (bone morphogenic protein) receptor 1B, and netrin 1 are required for targeting to and entry into the optic disc (Deiner et al., 1997; Birgbauer et al., 2000; Liu et al., 2003). In

\footnotetext{
Received March 30, 2006; revised June 19, 2006; accepted June 26, 2006.

This work was supported by The Wellcome Trust Grants 065606 and 072495 . We thank Dr. Marc Tessier-Lavigne for providing the slit knock-out mice used in this study. We also thank Prof. Glen Jeffery for his assistance with the retinal histology and Bennett Alakakone and Profs. Glen Jeffery and Carol Mason for their comments on this manuscript.

*H.T. and O.C. contributed equally to this work.

Correspondence should be addressed to Dr. Lynda Erskine, Institute of Ophthalmology, University College London, 11-43 Bath Street, London EC1V 9EL, UK. E-mail: I.erskine@ucl.ac.uk.

0. Camand's present address: Department of Neuroscience and Cell Biology, University of Medicine and Dentistry of New Jersey, Robert Wood Johnson Medical School, Piscataway, NJ 08854.

D. Barker's present address: Department of Anatomy and Developmental Biology, University College London, Gower Street, London WC1E 6BT, UK.

DOI:10.1523/JNEUROSCI.1342-06.2006

Copyright $\odot 2006$ Society for Neuroscience $\quad 0270-6474 / 06 / 268082-10 \$ 15.00 / 0$
}

chicks, graded expression of Sonic hedgehog across the retina plays a role in directing the central-peripheral growth of the axons (Kolpak et al., 2005). A number of cell adhesion molecules, including NCAM (neural cell adhesion molecule), NrCAM (NgCAM-related cell adhesion molecule), L1, and Neurolin/ DM-GRASP, also have been implicated in regulating intraretinal guidance (Brittis et al., 1995; Ott et al., 1998; Monnier et al., 2001; Zelina et al., 2005).

Slits are secreted guidance molecules that, acting through their Roundabout (Robo) receptors, mediate axon pathfinding (Brose et al., 1999; Li et al., 1999; Nguyen-Ba-Charvet et al., 1999, 2002; Bagri et al., 2002; Plump et al., 2002; Long et al., 2004; Sabatier et al., 2004). There are three Slits (Slit1-Slit3) in vertebrates (Holmes et al., 1998; Itoh et al., 1998; Brose et al., 1999; Li et al., 1999) and four Robos (Robo1-Robo4) (Kidd et al., 1998; Yuan et al., 1999a; Huminiecki et al., 2002), subsets of which are expressed in the developing retina. In rodents, from the time that the first RGCs are generated, both robo 2 and slit 1 are expressed in the RGC layer. Slightly later in development, slit2 also is expressed within the RGC and presumptive inner nuclear layers. In vitro, both Slit1 and Slit2 are potent inhibitors of RGC axon outgrowth (Erskine et al., 2000; Niclou et al., 2000; Ringstedt et al., 2000; Plump et al., 2002). Given the critical functions of the Slits in other regions of the optic pathway (Plump et al., 2002), these molecules appeared excellent candidates for factors mediating intraretinal axon guidance. Indeed, in chick retinas, alterations in Irx4 (iroquois homeobox protein 4) activity modulate slit1 expression and affect RGC axon fasciculation (Jin et al., 2003).

Using analyses of knock-out mice and in vitro assays, we found that, within dorsal retina exclusively, Slits control the initial po- 

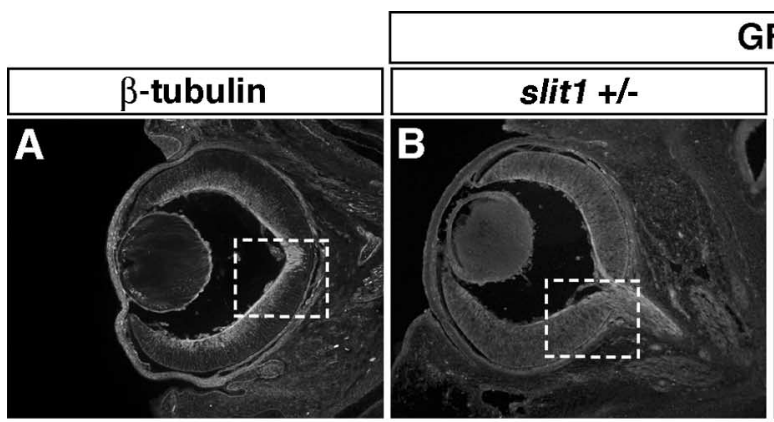

\section{GFP}
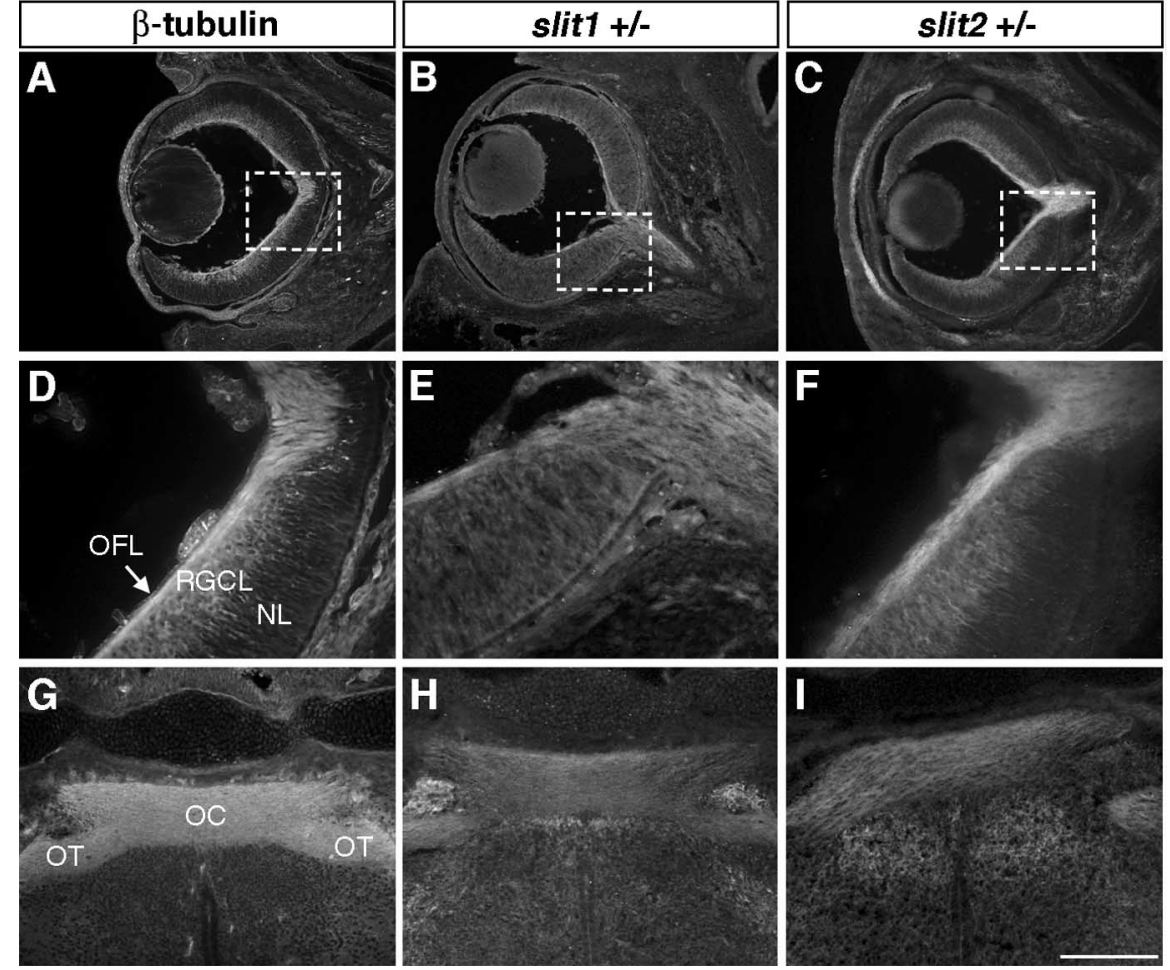

Figure 1. Determination of slit expression in the developing visual system using anti-GFP immunocytochemistry. Horizontal sections of E15.5 retina $(\boldsymbol{A}-\boldsymbol{F})$ and ventral diencephalon $(\boldsymbol{G}-\boldsymbol{I})$ stained with a neuron-specific anti- $\beta$-tubulin antibody to label the RGC axons $(\boldsymbol{A}, \boldsymbol{D}, \boldsymbol{G})$ or an anti-GFP antibody $(\boldsymbol{B}, \boldsymbol{C}, \boldsymbol{E}, \boldsymbol{F}, \boldsymbol{H}, \boldsymbol{I})$. Boxed regions in $\boldsymbol{A}-\boldsymbol{C}$ are shown at higher power in $\boldsymbol{D}-\boldsymbol{F}$. In both slit1 $^{+/-}(\boldsymbol{B}, \boldsymbol{E}, \boldsymbol{H})$ and slit2 ${ }^{+/-}(\boldsymbol{C}, \boldsymbol{F}, \boldsymbol{I})$ embryos, tau-GFP is expressed by RGC axons within the OFL at the inner surface of the retina $(\boldsymbol{B}, \boldsymbol{C}, \boldsymbol{E}, \boldsymbol{F})$ and in the optic nerve $(\boldsymbol{B}, \boldsymbol{C}, \boldsymbol{E}, \boldsymbol{F})$, chiasm $(\boldsymbol{H}, \boldsymbol{I})$, and tract $(\boldsymbol{H}, \boldsymbol{I})$. Expression is also present in specific regions of the diencephalon surrounding the developing optic chiasm $(\boldsymbol{H}, \boldsymbol{I})$ and, in slit2 ${ }^{+/-}$embryos, the cornea and lens epithelium $(\boldsymbol{C})$. NL, Neuroblastic layer; OC, optic chiasm; RGCL, RGC layer; 0T, optic tract. Scale bar: A-C, $400 \mu \mathrm{m} ; \mathbf{D}-\boldsymbol{F}, 100 \mu \mathrm{m} ; \mathbf{G}-\mathbf{I}, 200 \mu \mathrm{m}$.

larity of RGC axon outgrowth and prevent a subset of RGC axons located predominately in ventral retina from straying away from the OFL. Although disorganized or displaced, the aberrant axons within both dorsal and ventral regions exit the eye. These data add Slits to the growing list of factors that regulate intraretinal pathfinding and demonstrate a surprising degree of regional specificity in their mode of action.

\section{Materials and Methods}

Embryos. All conditions and experimental procedures were in accordance with the United Kingdom Animals (Scientific Procedures) Act 1986 and associated guidelines. Experiments were performed using wildtype (WT) C57BL/6J or slit-deficient mice (Plump et al., 2002) maintained in timed-pregnancy breeding colonies. Noon on the day on which a plug was found was considered embryonic day 0.5 (E0.5). Pregnant mothers were killed using a rising gradient of $\mathrm{CO}_{2}$, and the embryos were removed by cesarean section. Embryos were genotyped by PCR as described previously (Plump et al., 2002) and fixed overnight in $4 \%$ paraformaldehyde in PBS or, for histological analysis, perfused with $2 \%$ paraformaldehyde $/ 2 \%$ glutaraldehyde in PBS, followed by overnight fixation in the same solution. Adult animals were killed and perfusion fixed in the same way.

Immunostaining, DiI labeling, and histology. For immunocytochemistry on tissue sections, embryonic heads were cryoprotected with $25 \%$ sucrose, embedded in OCT (RA Lamb, Eastbourne, UK), and sectioned horizontally at $14 \mu \mathrm{m}$ on a cryostat. Sections were collected on SuperFrost Plus slides (VWR International, Lutterworth, UK) and blocked with $10 \%$ goat serum (NGS)/0.2\% Triton X-100/PBS, followed by overnight incubation in primary antibody diluted in the blocking solution.
The following antibodies were used: rabbit anti-green fluorescent protein (GFP) (1:500; Invitrogen, Paisley, UK), TUJ1 (anti-neuronspecific- $\beta$-tubulin) (1:1000; Cambridge Bioscience, Cambridge, UK), anti-phosphohistone H3 (1:100; Upstate Biotechnology, Lake Placid, NY), 39.4D5 (anti-Islet1; 1:50; Developmental Studies Hybridoma Bank, University of Iowa, Iowa City, IA), and anti-calretinin (1:10,000; Swant, Bellinzona, Switzerland). The sections were washed with PBS, incubated for $2 \mathrm{~h}$ with goat anti-rabbit IgG-cyanine 3 (Cy3) ( $1: 500$ in $1 \%$ NGS/PBS; Jackson ImmunoResearch, Soham, UK), goat anti-mouse IgG-Cy3 (1:500; Jackson ImmunoResearch), or AlexaFluor-488 goat anti-mouse IgG (1:200; Invitrogen), followed by additional washes, and mounted with Vectashield (Vector Laboratories, Peterborough, UK). Control sections from wild-type animals or from which the primary antibody was omitted showed no staining (data not shown).

Before removing whole retinas for labeling, a cut was made in the nasal pole to enable orientation, and the lens was removed. Retinas were blocked as above and incubated in either mAb3A10 (anti-neurofilament; 1:200; Developmental Studies Hybridoma Bank) or TUJ1, followed by goat anti-mouse IgG-Cy3 (1:2000) or AlexaFluor-488 goat anti-mouse IgG (1:200). Labeled retinas were flat mounted, OFL side up, or embedded in 3\% agarose, sectioned at $100 \mu \mathrm{m}$ on a vibratome, and mounted using ProLong Gold (Invitrogen).

For retrograde labeling of RGCs, the diencephalon was dissected free from overlying tissue, and a large crystal of DiI (Invitrogen) was placed into the optic chiasm. The tissue was left in PBS plus $0.1 \%$ sodium azide at $37^{\circ} \mathrm{C}$ for $2 \mathrm{~d}$, and the labeled retinas were removed as above and either flat mounted or sectioned at $100 \mu \mathrm{m}$ on a vibratome and mounted in Vectashield.

For histological analysis, embryonic and adult retinas were dehydrated and embedded in Technovit 7100 (TAAB Laboratories Equipment, Aldermaston, UK) and sectioned coronally at $5 \mu \mathrm{m}$ on a microtome. The sections were stained briefly with cresyl violet and dehydrated and mounted in DPX (VWR International).

Labeled sections and retinas were photographed using an Olympus Optical (Tokyo, Japan) BX50 microscope and a Nikon (Tokyo, Japan) DXM1200 digital camera with ACT-1 software or using a Zeiss (Oberkochen, Germany) LSM 510 confocal microscope. Images were prepared using Adobe Photoshop (Adobe Systems, San Jose, CA).

Collagen gel cultures. Retinal explants taken from the peripheral region of E15.5 wild-type embryos were cultured in collagen gels either alone or with lenses from E15.5 wild-type, sli1 ${ }^{-/-}$, slit $1^{-/-} ;$slit2 ${ }^{+/-}$, or slit1/2 $2^{-/-}$ embryos essentially as described previously (Erskine et al., 2000). In a separate series of experiments, explants taken from each quadrant of E14.5 WT retinas were cocultured with clusters of Slit1-expressing cells (Plump et al., 2002). After 18-24 h, the cultures were fixed with $4 \%$ paraformaldehyde in PBS and stained with an anti- $\beta$-tubulin antibody (1:500; Sigma-Aldrich, Gillingham, UK), followed by a goat anti-mouse IgG-Cy3 secondary antibody (1:2000) to reveal the extent of RGC axon outgrowth. Labeled cultures were photographed using a Nikon SMZ 1500 fluorescent microscope and DXM1200 digital camera, and the extent of axon outgrowth was quantified as described previously (Erskine et al., 2000) by using the public domain NIH Image program to measure the area covered by the RGC axons. This measurement takes into account both the length and the number of the axons. The cultures were set up and analyzed blind to the genotype of the lenses, and the results are the 
mean \pm SEM of four independent experiments. Statistical comparisons were performed using student's unpaired $t$ test.

\section{Results}

\section{slit 1 and slit 2 are expressed by RGCs}

Using in situ hybridization both slit1 and slit2 have been demonstrated previously to be expressed in the inner region of the developing rodent retina, although the precise identity of the expressing cells remains controversial (Yuan et al., 1999b; Erskine et al., 2000; Niclou et al., 2000; Ringstedt et al., 2000; Jin et al., 2003). To clarify the identity of the slit-expressing cells, we made use of the fact that the slit-deficient mice express tau-GFP under the control of the endogenous slit1 or slit2 promoters (Plump et al., 2002). Consequently, any cells that normally express slit1 or slit2 mRNA now express GFP in their cell bodies and processes.

In both slit $^{+/-}$and slit $^{+/-}$embryos, tau-GFP is expressed in the inner region of the retina, including the OFL and optic nerve (Fig. $1 B, C, E, F$ ), in a pattern that correlates with the location of the RGCs and their axons (Fig. $1 A, D)$. In slit $2^{+/-}$ embryos tau-GFP also is expressed in the cornea and lens epithelium (Fig. 1C). In the ventral diencephalon of both genotypes, tau-GFP is expressed in the optic chiasm and tracts and in specific domains bordering the optic pathway (Fig. $1 G-I$ ). In addition to RGC axons (Fig. $1 G$ ), the only other cell types present at the chiasmatic midline are radial glial cells and an early born population of diencephalic neurons, both of which have distinct morphologies and arrangements from the RGC axons (Marcus et al., 1995; Erskine et al., 2000). These data confirm the expression patterns obtained using in situ hybridization (Yuan et al., 1999b; Erskine et al., 2000; Niclou et al., 2000; Ringstedt et al., 2000) and demonstrate clearly that, in the mammalian retina, both slit1 and slit2 mRNA are expressed by RGCs.

\section{Slits help restrict RGC axons to the OFL}

To establish whether Slits are critical for axon guidance in the mammalian retina, we stained E16.5 wild-type or slit-deficient retinas with a neuron specific anti- $\beta$-tubulin or antineurofilament antibody to label all of the RGC axons and viewed them using confocal microscopy in flat mounts or coronal sections (Fig. 2).

In wild-type retinas, RGC axons are restricted to the OFL at the inner surface of the retina, and in only 1 of 20 retinas was a single bundle of axons seen within the outer (RGC and neuroblastic) layers (Figs. $2 B, F, 3 A, C$ ). In mice lacking slit1 alone, RGC axons remained restricted to the OFL (Figs. 2C, G, 3C). However, in slit2-deficient and, more frequently, slit1/2-deficient retinas, a subset of RGC axons extended away from the OFL into
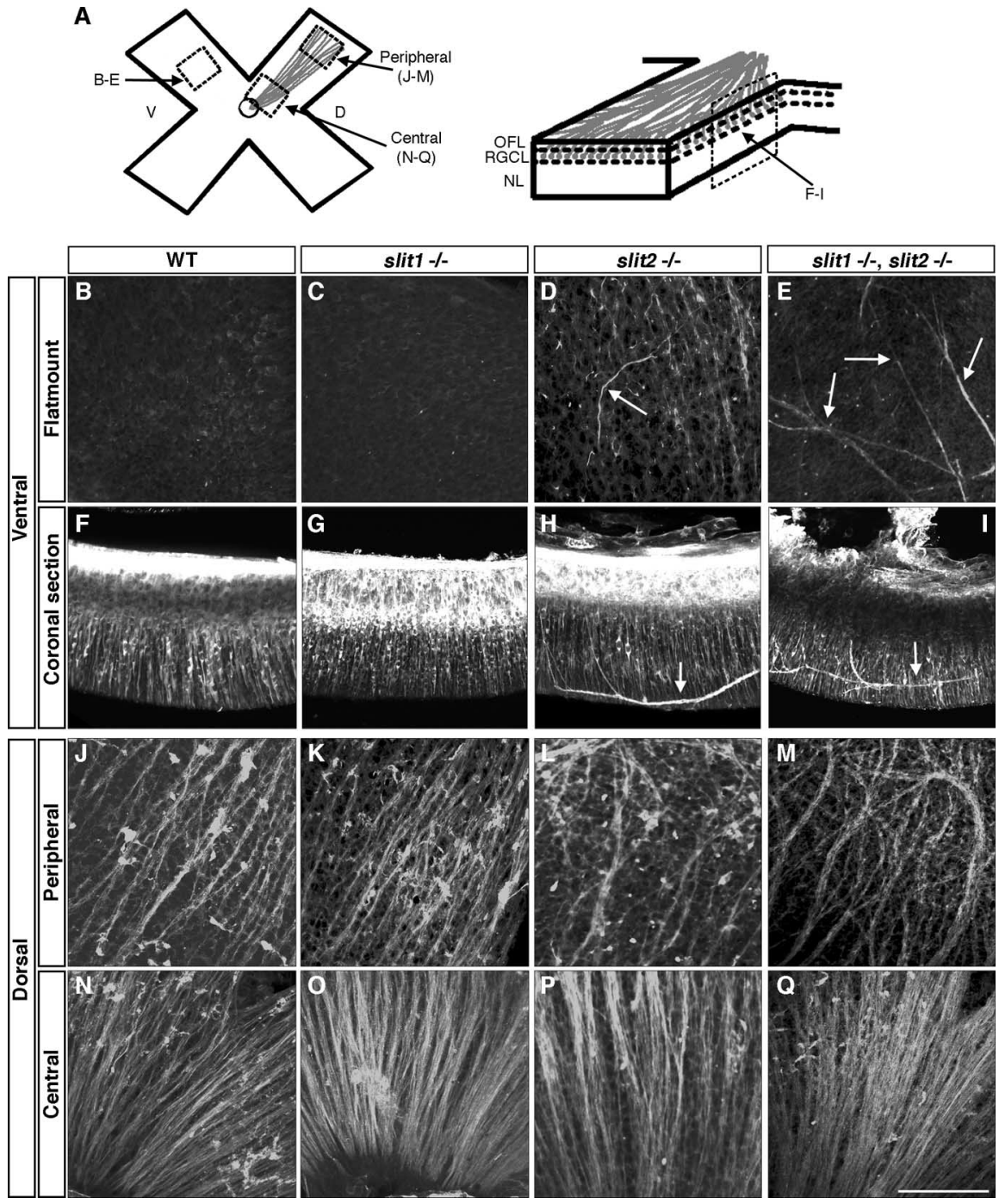

Figure 2. Slits regulate intraretinal axon guidance. $A$, Schematic diagram illustrating the regions of the retina imaged and the normal organization of the retina. D, Dorsal; V, ventral; RGCL, RGC layer; NL, neuroblastic layer. $\boldsymbol{B}-\boldsymbol{E}$, J-Q, Confocal images taken at the level of the ventral neuroblastic layer $(\boldsymbol{B}-\boldsymbol{E})$ or dorsal OFL $(\boldsymbol{J}-\mathbf{Q})$ of flat-mounted E16.5 retinas stained with an anti- $\beta$ anti- $\beta$-tubulin-stained E16.5 retinas. In each image, the optic disc is located toward the right. In wild-type $(\boldsymbol{B}, \boldsymbol{F}, \boldsymbol{J}, \boldsymbol{N})$ and 1-deficient $(\boldsymbol{C}, \mathbf{G}, \boldsymbol{K}, \mathbf{0})$ retinas, $\mathrm{RGC}$ axons extend directly toward the optic $\operatorname{disc}(\boldsymbol{J}, \boldsymbol{K}, \boldsymbol{N}, \mathbf{0})$ and are restricted to the $0 \mathrm{FL}(\boldsymbol{B}, \boldsymbol{C}, \boldsymbol{F}$ (arrows). The organization of the dorsal OFL also is perturbed, with many axons within the peripheral $(\boldsymbol{L}, \boldsymbol{M})$ but not central $(\boldsymbol{P}, \mathbf{Q})$ region adopting abnormal looped trajectories. Scale bar: $\boldsymbol{B}-\boldsymbol{E}, \boldsymbol{J}-\mathbf{Q}, 100 \mu \mathrm{m} ; \boldsymbol{F}-\mathbf{I}, 125 \mu \mathrm{m}$.

the outer retina. These axons originated from cells located within the RGC layer and formed highly fasciculated bundles that, although less directed than axons within the OFL, extended in the overall direction of the optic disc (Figs. $2 D, E, H, I, 3 B$ ). An average of five bundles of RGC axons were located within the outer layers of slit2-deficient retinas increasing to over 30 bundles in the double mutants (Fig. $3 C$ ). There also was a dorsoventral polarity in the location of the ectopic axons. In both slit2- and slit1/ 2-deficient retinas, more than twice as many RGC axon bundles extended through the outer layers of ventral than dorsal retina (Fig. $3 B, D)$.

\section{Slits regulate the organization of the OFL}

Slits also are involved in regulating the directed growth of RGC axons within the OFL itself (Fig. 2J-Q). In wild-type retinas, RGC axons originating throughout the retina extended within the OFL 

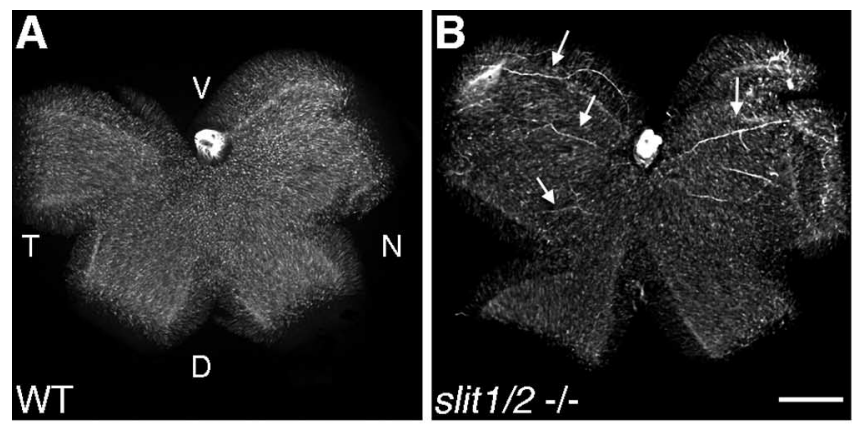

\section{C}

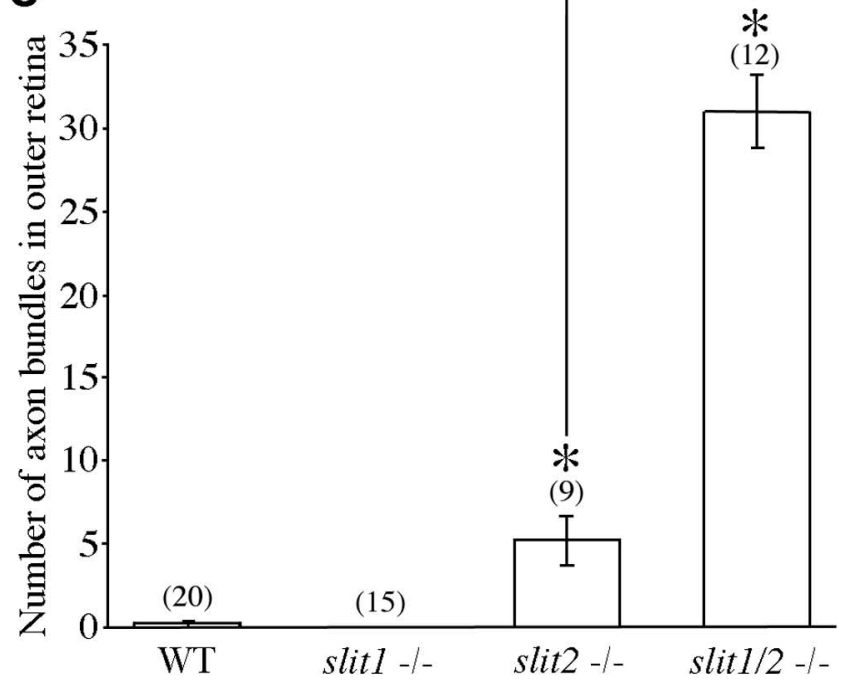

D

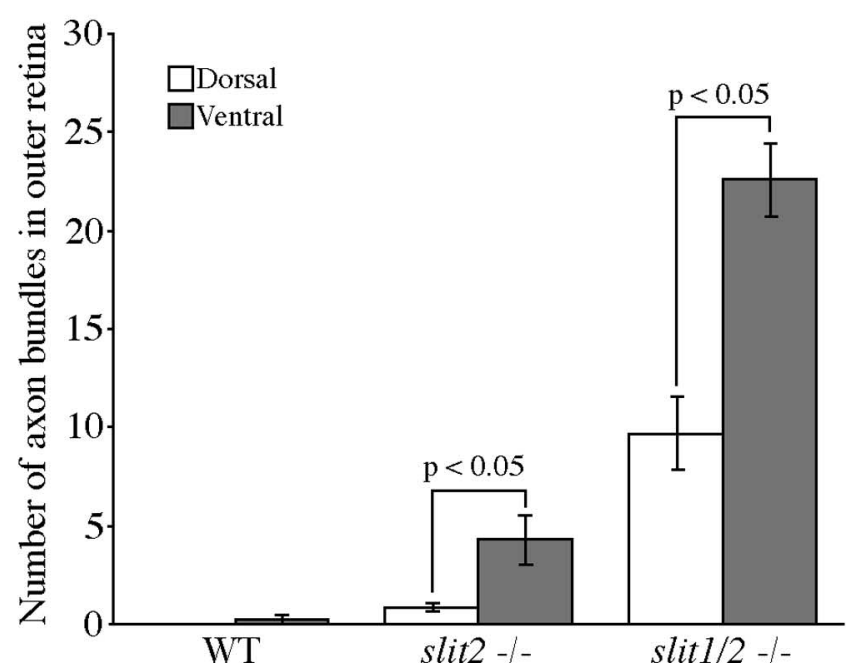

Figure 3. Spatial organization and quantification of number of RGC axon bundles in the outer layers of slit-deficient retinas. $\boldsymbol{A}, \boldsymbol{B}$, Confocal images of flat-mounted E14.5 wild-type $(\boldsymbol{A})$ and slit1/2-deficient $(\boldsymbol{B})$ retinas at the level of the outer retina. In slit1/2-deficient retinas $(\boldsymbol{B})$ but not wild-type retinas $(\boldsymbol{A})$, a subset of RGC axons originating predominately within ventral retina are present within the outer layers of the retina. D, Dorsal; N, nasal; T, temporal; $V$, ventral. Scale bar, $200 \mu \mathrm{m}$. C, Mean \pm SEM number of axon bundles located within the outer retina of wild-type, slit1-, slit2-, and slit1/2-deficient retinas. ${ }^{*} p<0.001$ compared with wildtype. $\boldsymbol{D}$, Mean \pm SEM number of axon bundles in the outer layers of dorsal (white bars) or ventral (gray bars) retina. In both slit2- and slit1/2-deficient retinas, significantly more bundles are found within the outer layers of ventral versus dorsal retina. in a straight, directed manner toward the optic disc. Polarity in the direction of growth was evident from the outset with the axons originating from the side of each cell closest to the optic disc and extending directly toward this region (Fig. 2 J, N). Surprisingly, in slit1-deficient retinas, we found no obvious changes in the extent of RGC axon fasciculation or the organization of the OFL (Fig. $2 \mathrm{~K}, \mathrm{O}$ ). However, in mice lacking slit 2 or slit1/2, the organization of the OFL was perturbed, with many RGC axons originating in the periphery of the retina deviating from their normal peripheral-central orientation and forming abnormal looped and curved trajectories (Fig. $2 L, M$ ). This was primarily attributable to the axons initially growing toward the retinal periphery before correcting their direction of growth and extending toward the optic disc. Excessive crossing of axons between different fascicles also occurred (Fig. $2 L, M$ ). Surprisingly, these pathfinding errors were found exclusively within dorsal retina in which they were restricted to the peripheral part of the retina (Fig. $2 L, M, P, Q)$. Because of the fact that we labeled all RGC axons, we were unable to quantify the precise number of axons with aberrant trajectories. However, in both slit2 and slit1/2-deficient mice, the guidance of most, if not all, RGC axons originating in the most peripheral 25\% of dorsal retina (Fig. $4 M$ ) was affected, whereas more centrally the orderly projection of RGC axons toward the optic disc occurred normally (Fig. 2P,Q). The organization of the OFL within the entire ventral half of the retina also was normal (supplemental Fig. 1, available at www.jneurosci.org as supplemental material). Within the dorsal retina, there was no difference in the severity of the OFL defects between the slit2-and slit1/2-deficient retinas, demonstrating that Slit2 alone regulates this aspect of intraretinal guidance.

\section{Development of intraretinal pathfinding defects in slit-deficient mice}

These two defects in intraretinal pathfinding, the projection of RGC axons into the outer layers of the retina and the disorganization of the OFL, develop over different time courses. At E13.5, the organization of the OFL in the slit1/2-deficient mice was normal, whereas significant numbers of axons projected away from the OFL into the outer retina (Fig. $4 A-D$ ). From E14.5 onward, both the organization of the OFL and the restriction of RGC axons to the OFL was perturbed (Fig. $4 E-L$ ). Furthermore, at all ages, the regional specificity of Slits function in the retina was maintained, with the disorganization of the OFL occurring exclusively in dorsal retina and approximately twice as many axon bundles projecting into the outer layers of ventral than dorsal retina (Fig. 4C-L) (supplemental Fig. 1, available at www. jneurosci.org as supplemental material). This demonstrates clearly that the differential requirement for Slits within ventral and dorsal retina is not the result of a ventrodorsal gradient in retinal maturity (Halfter et al., 1985).

As the retina develops, new cells are added at progressively more peripheral locations, resulting in a central-to-peripheral expansion of the neuroepithelium. As a result, what was peripheral retina at E14.5 is now located more centrally at later stages of development (Fig. 4M). Despite this, at all ages, the disorganization of the OFL in the slit1/2-deficient mice occurred exclusively within the peripheral $22-25 \%$ of the dorsal retina (Fig. $4 M$ ). More centrally and within ventral retina, axon organization was indistinguishable from that seen in age matched wild-type retinas (Fig. 2N-Q) (supplemental Fig. 1, available at www.jneurosci.org as supplemental material). This suggests that, in dorsal but not ventral retina, Slits act to control the initial outgrowth of newly 
differentiated RGCs, and, as development proceeds, the aberrant trajectory of these axons is corrected.

\section{In slit-deficient retinas, displaced and disorganized RGC axons exit the optic disc}

To establish whether the RGC axons displaced from the OFL or with aberrant initial trajectories subsequently exit the retina, we placed a crystal of DiI at the optic chiasm to retrogradely label only those axons that have extended out of the eye (Fig. 5A). At both E16.5 and E18.5, guidance errors similar to those seen in the antibody-stained retinas were found (Fig. 5 and data not shown). After retrograde labeling, in slit1/2-deficient retinas, but not their slit1-deficient littermates, RGC axons with aberrant initial trajectories were found exclusively within the peripheral 25\% of the dorsal OFL (Fig. 5B,C) and extending through the outer layers the retina (Fig. $5 D, E$ ). This demonstrates that, although the location or initial trajectory of some RGC axons is perturbed, they are still able to navigate to the optic disc and exit the retina.

\section{Retinal morphology is normal in slit-deficient mice}

One explanation for these pathfinding defects is that they occur secondarily to changes in the overall structure of the retina. We therefore examined the gross morphology and lamination of the slitdeficient retinas. We found that, in slit1and/or slit2-deficient embryos, the overall size and shape of the retinas was normal and that the optic fissure closed completely (data not shown). In thin sections stained with cresyl violet to reveal the ar-

rangement and relative size of the various retinal layers, we also found no defects in retinal organization (Fig. 6A-F). At E18.5, wild-type retinas are composed of three layers: the OFL at the inner surface of the retina, an inner differentiated region, and an outer neuroblastic region (Fig. 6A). In both slit1- and slit1/2deficient retinas, we found no differences in the organization or thickness of these layers (Fig. $6 B, C$ ). The number of mitotic and pyknotic cells also was not significantly different from that found in wild-type retinas (data not shown).

Mice lacking slit2 die at birth, and therefore we were unable to assess retinal development in these mice or the double knockouts at later stages of development. However, in adult slit1deficient retinas, we found that all of the different retinal layers were present and were of similar organization and thickness to wild-type or slit $1^{+/-}$retinas (Fig. $6 D-F$ ).

We stained sections of E16.5 retinas with cell-type-specific markers to determine whether in the slit-deficient mice the major classes of retinal cells are present and in their proper places. In both WT and slit $1 / 2^{-/-}$retinas, mitotic cells, labeled with an antibody against phospho-histone $\mathrm{H} 3$, were located exclusively adjacent to the retinal pigment epithelium (Fig. 6G,J). In con- trast, in both genotypes, Islet1- and calretinin-positive differentiated RGCs and amacrine cells were localized to the inner region of the retina (Fig. $6 H, I, K, L$ ). Together, this strongly suggests that the intraretinal guidance defects are attributable to pathfinding errors rather than changes in the gross structure of the retina or the misdifferentiation or misplacement of retinal components.

In vitro Slits are secreted by the lens and inhibit outgrowth of dorsal RGC axons

Although slit2 is expressed throughout the entire centralperipheral axis of the retina (Fig. 1) (Erskine et al., 2000), it is required only within the most peripheral $25 \%$ of dorsal retina for normal OFL formation (Figs. 2, 4). Our working model is that this reflects release of Slit2 from nonretinal ocular tissues. An excellent candidate for this source of Slit is the developing lens. The lens is ideally situated within the developing eye to preferentially influence the outgrowth of axons located within the retinal periphery (Figs. 1, 5F). Furthermore, the lens expresses Slit2 (Fig. 1) (Yuan et al., 1999b; Erskine et al., 2000; Niclou et al., 2000), and, in vitro, diffusible factors secreted by the lens inhibit outgrowth of chick RGC axons (Ohta et al., 1999). 
A
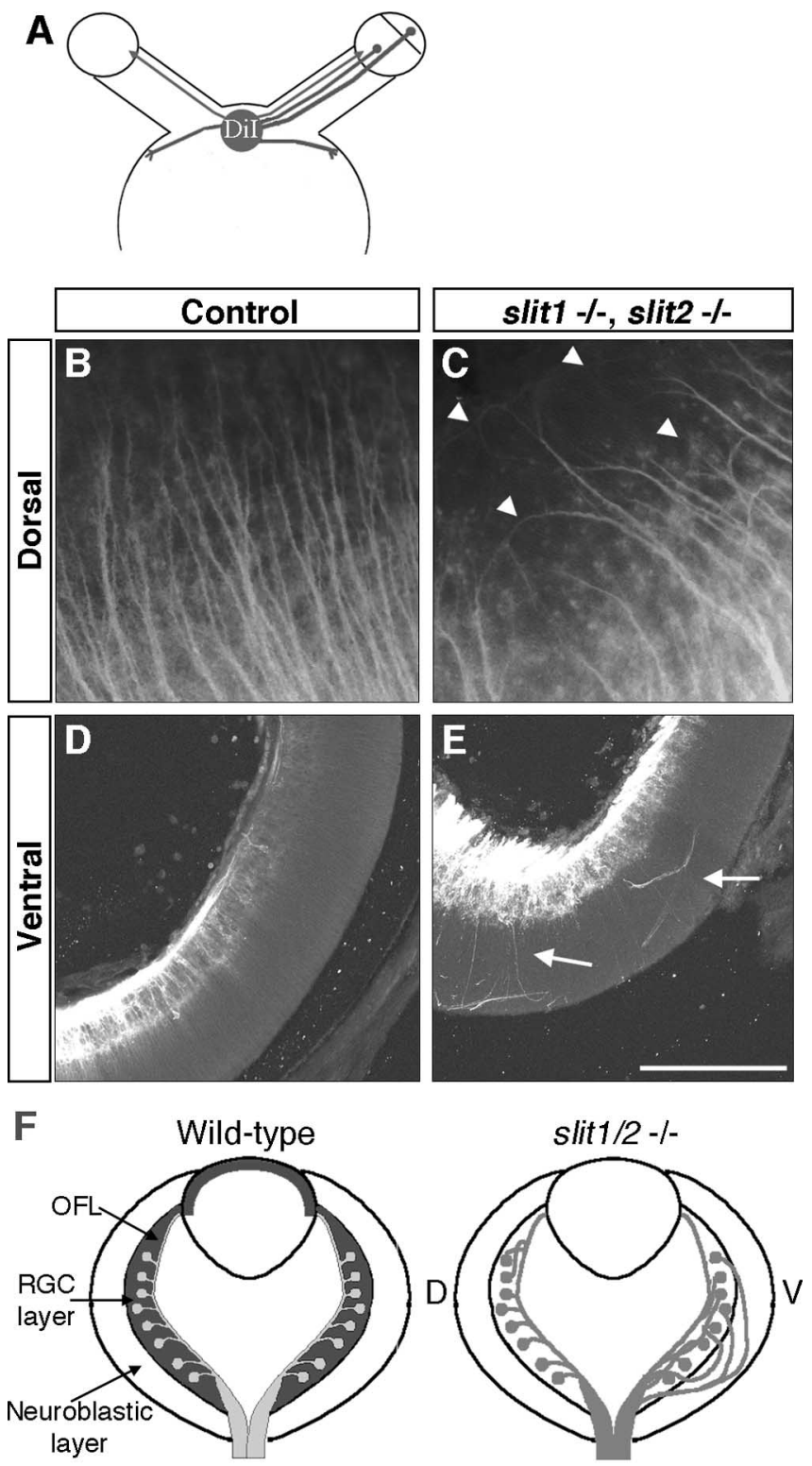

slit $1 / 2$

Figure 5. The aberrantly organized RGC axons exit the optic disc. $\boldsymbol{A}$, Schematic diagram illustrating the labeling method. A crystal of Dil was applied to the optic chiasm and retrogradely labeled only those axons that have extended into this region. $\boldsymbol{B}-\boldsymbol{E}, \mathrm{E} 18.5$ dorsal $(\boldsymbol{B}, \boldsymbol{C})$

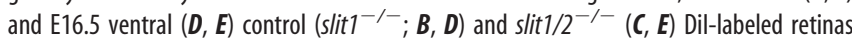
viewed as flat mounts $(\boldsymbol{B}, \boldsymbol{C})$ or after sectioning coronally $(\boldsymbol{D}, \boldsymbol{E})$. In each image, the direction of the optic disc is toward the bottom of the picture. In control retinas $(\boldsymbol{B}, \boldsymbol{D})$, all RGC axons are restricted to the $\mathrm{OFL}$ in which they extend directly toward the optic disc. In slit $1 / 2^{-1-}$ retinas, abnormally organized axons within the peripheral dorsal retina (arrowheads in $\boldsymbol{C}$ ) are labeled, as are a significant number of axons within the outer layers of ventral retina (arrows in $E$ ). Scale bar: $\boldsymbol{B}, \boldsymbol{C}, 125 \mu \mathrm{m} ; \boldsymbol{D}, \boldsymbol{E}, 250 \mu \mathrm{m}$. $\boldsymbol{F}$, Summary of intraretinal pathfinding defects in slit-deficient mice. RGC axons extend normally into the OFL at the inner surface of the retina in which they grow in a highly directed manner toward the optic disc. In slit-deficient retinas, the initial direction of growth from recently differentiated RGCs located exclusively within dorsal retina is perturbed, and a subset of axons originating predominately in ventral retina stray away from the $\mathrm{OFL}$ into the outer layers of the retina. These displaced or disorganized axons ultimately exit the optic disc. D, Dorsal; $V$, ventral.

To test whether loss of slit 2 from the lens potentially underlies the defects in OFL organization, we cultured E15.5 wild-type retinal explants from peripheral dorsotemporal or ventrotemporal retina either alone or with lenses from age-matched wild-type or slit-deficient embryos (Fig. 7A-E). Lenses from WT, slit1 ${ }^{-/-}$, and $s$ lit $1^{-/-} ;$slit $2^{+/-}$embryos had a potent inhibitory effect on outgrowth from dorsal retinal explants, inducing an $\sim 40 \%$ decrease compared with that from explants cultured alone (Fig. $7 A, B, D)$. However, in cultures containing slit1/2-deficient lenses, significantly more outgrowth occurred than in cultures containing lenses of the other genotypes (Fig. $7 A-D$ ). Some inhibition of outgrowth still occurred, however, with the slit1/2deficient lenses inducing an $\sim 20 \%$ decrease in the extent of outgrowth seen in the absence of the lens (Fig. 7D). This strongly suggests that Slit2 is a component of the inhibitory activity secreted by the developing lens. In contrast, both WT and slitdeficient lenses had only a weak inhibitory effect on outgrowth from ventral retinal explants, inducing only an $\sim 20 \%$ decrease in the extent of axon outgrowth. Furthermore, no difference was seen in the potency of the tissues tested (Fig. 7E).

\section{In vitro, Slit1 inhibits outgrowth of both dorsal and ventral RGC axons}

We have shown previously that RGC outgrowth from explants taken from both dorsal and ventral regions of the retina is inhibited by Slit2 (Erskine et al., 2000). To test whether this is also true for Slit1, we cocultured explants taken from each quadrant of WT retinas with clusters of either mock- or Slit1-transfected cells. Slit1 had a potent inhibitory effect on outgrowth from explants taken from each quadrant of the retina (Fig. $7 F$ ) and induced a similar percentage decrease in the extent of axon outgrowth from both ventral and dorsal regions (Fig. 7G). This demonstrates that the regional specificity of Slit function in the retina is unlikely to be attributable to intrinsic differences in the responsiveness of dorsal and ventral RGC axons to Slits.

\section{Discussion}

Surprisingly, we found that Slits regulate distinct aspects of RGC axon guidance within different regions of the retina. In ventral retina, Slits act specifically to help restrict RGC axons to the OFL, whereas in dorsal retina, they function predominately to regulate the ordered growth of RGC axons within the OFL layer itself (Fig. $5 F)$. This is despite the expression of Slits throughout the retina and the ability of dorsal and ventral RGCs to mediate Slitsignaling (Erskine et al., 2000). We also identified the lens as a potential source of Slit regulating the initial polarity of outgrowth within the dorsal OFL. However, in the absence of Slits, axons that are displaced from the OFL or have initially disorganized trajectories can still exit the eye. These data demonstrate clearly that multiple mechanisms exist in the retina for axon guidance, of which Slits are an important component.

\section{Slits regulate distinct aspects of intraretinal guidance in the mammalian and chick retina}

Although Slit1 is expressed in both the murine and chick retina, its localization and function are not conserved. In chicks, slit1 is expressed by amacrine cells and plays an important role in controlling the fasciculation of RGC axons as they extend toward the optic disc (Jin et al., 2003). In contrast, we found that, in mouse, both slit1 and slit2 are expressed predominately, if not exclusively, by RGCs and that loss of slit1 alone has no effect on intraretinal pathfinding. Other guidance cues, for example, CSPGs, also have different patterns of expression in rodent and chick retinas. In rats, CSPGs are dynamically expressed peripheral to recently differentiated RGCs and, by acting as an inhibitory guidance cue, help prevent RGC axons from extending into the retinal periphery (Brittis et al., 1992). In contrast, in chicks, CSPGs are 
located in regions in which RGCs extend actively (McAdams and McLoon, 1995; Ring et al., 1995). Together, this suggests that fundamentally distinct mechanisms may regulate intraretinal axon guidance in the mammalian and chick retina.

\section{Slits help prevents RGC axons from extending into the outer layers of the retina}

In the absence of Slits, a subset of RGC axons project aberrantly through the outer layers of the retina. Although we cannot exclude that Slits act normally to attract RGC axons toward the OFL (Jin et al., 2003), our preferred model is one in which they function in an inhibitory manner to prevent RGC axon extension away from this region. In vitro, both Slit 1 and Slit 2 are potent inhibitors of RGC axon outgrowth, and their expression within the RGC and presumptive inner nuclear layers of the retina place them in an ideal location to act as a barrier to RGC axon extension (Erskine et al., 2000; Niclou et al., 2000; Ringstedt et al., 2000; Plump et al., 2002). Furthermore, because removal of preexisting axons from the OFL is not sufficient to induce ectopic projection of RGC axons into the outer layers of the retina (Stier and Schlosshauer, 1995), loss of Slit from the OFL alone is unlikely to be responsible for the observed guidance defects.

Although displaced from the OFL, the mislocalized axons project through the outer retina in a highly directed manner toward the optic disc. This demonstrates that the cues that direct axons toward the optic disc are not localized exclusively within the OFL (Goldberg, 1977), and signaling molecules other than Slits, for example, Sonic hedgehog and downstream targets of Zic3 (zic family member 3 heterotaxy 1) (Zhang et al., 2004; Kolpak et al., 2005), are key regulators of this aspect of intraretinal guidance. Furthermore, as they extend through the outer retina, the displaced axons form tightly fasciculated bundles, demonstrating that the outer retina is still partially inhibitory to RGC axon outgrowth. This suggests that other guidance cues function cooperatively with Slits to prevent extension into the outer retina, and redundancy between these cues is the simplest explanation for why in the absence of Slits the majority of axons remain restricted to the OFL.

\section{Slit 2 controls the initial direction of RGC axon outgrowth}

Newly differentiated RGCs extend transient processes in random directions until ultimately a process originating from the side of the cell closest to the optic disc becomes stabilized and forms the mature axon (Brittis and Silver, 1995). We identified Slit2 as a key factor regulating the initial polarity of outgrowth from dorsal but not ventral retina. Furthermore, we found that the lens, a tissue known to be inhibitory to RGC axon outgrowth (Ohta et al., 1999), secretes Slit2. Because of its position in the eye, signals from the lens will influence preferentially RGCs located in the retinal periphery (Fig. $5 F$ ). Thus, we propose that it is this extrinsic source of Slit that regulates the initial polarity of RGC axon outgrowth within dorsal retina.

Slit2 alone, however, is not a critical factor in determining the overall growth of RGC axons toward the optic disc, and other factors ultimately compensate for its loss. Signals from the lens in combination with inhibitory guidance molecules, such as CSPGs within the extreme periphery of the retina (Brittis et al., 1992; Brittis and Silver, 1995), and attractive cues emanating from more central regions (Kolpak et al., 2005) may underlie this corrected growth.

There are striking similarities in the functions of Slit2 and CSPGs in directing intraretinal pathfinding, with both factors acting to control the initial polarity of RGC axon outgrowth and prevent extension into the retinal periphery (Brittis et al., 1992; Brittis and Silver, 1995). Proteoglycans are key regulators of guid- 

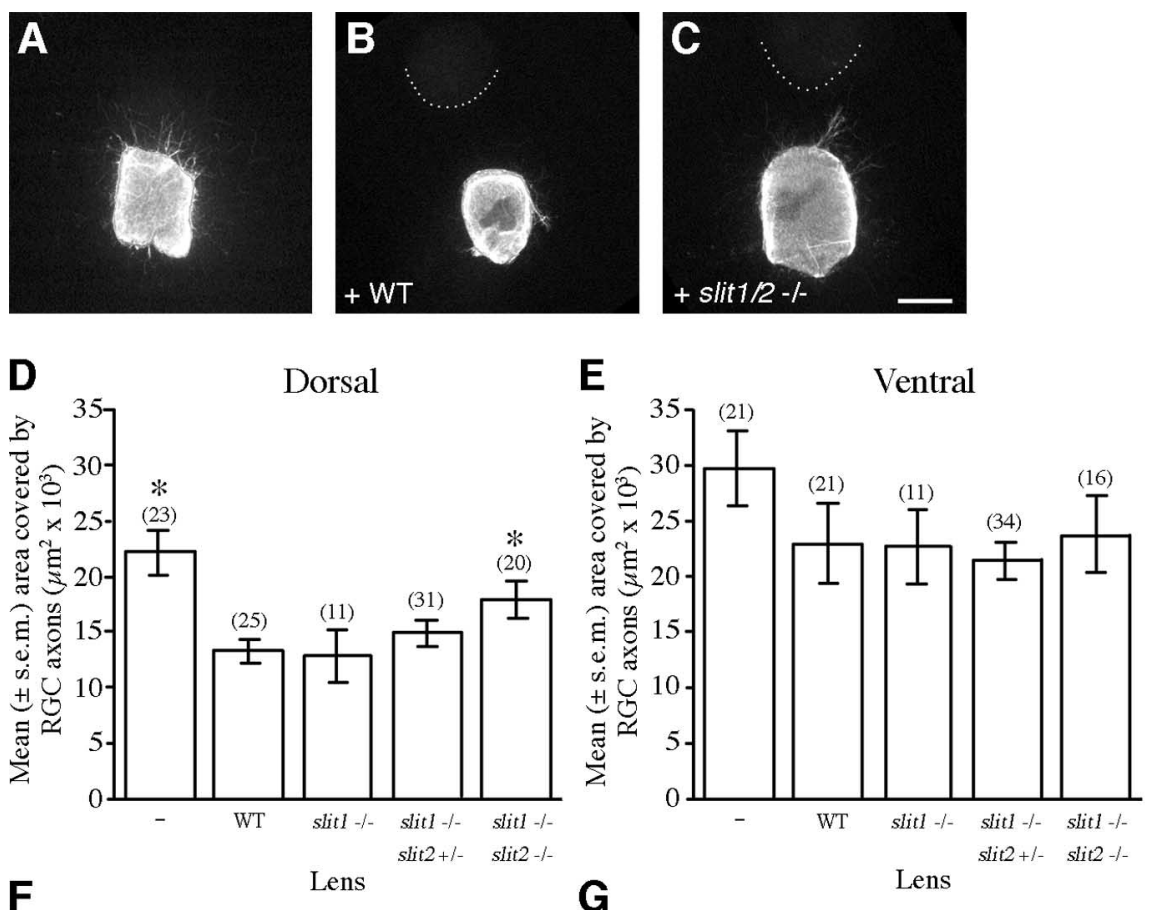

$\mathbf{F}$
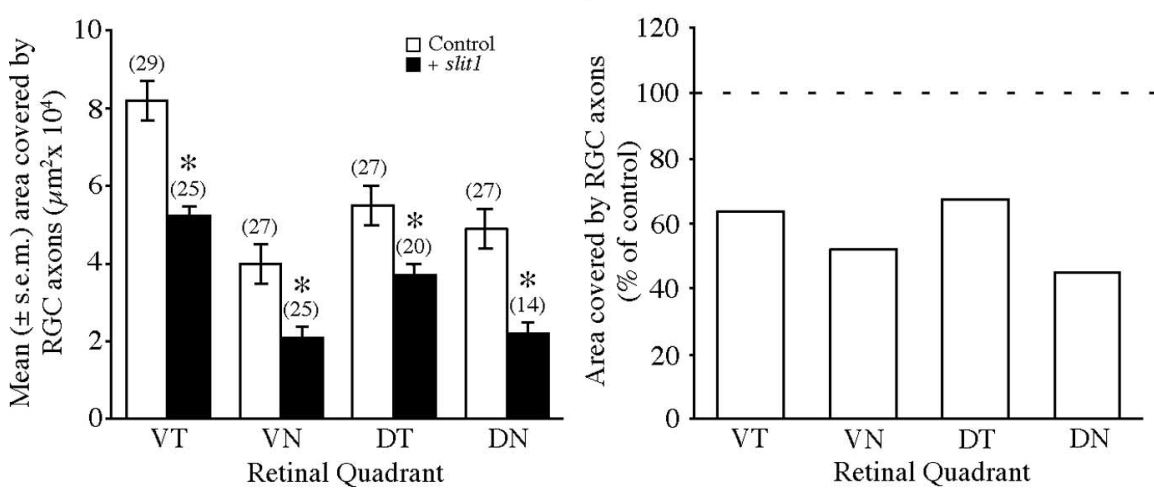

Figure 7. Slit proteins from the lens inhibit RGC axon outgrowth from dorsal retinal explants. $\boldsymbol{A}-\boldsymbol{C}$, Representative examples of E15.5 dorsal retinal explants cultured alone $(\boldsymbol{A})$ or with an E15.5 wild-type $(\boldsymbol{B})$ or slit1/2-deficient $(\boldsymbol{C})$ lens. Significantly more outgrowth occurs in the presence of the slit-deficient lens than the wild-type lens. Scale bar, $250 \mu \mathrm{m}$. D, E, Quantification of the extent of axon outgrowth in cocultures of dorsal $(\boldsymbol{D})$ or ventral $(\boldsymbol{E})$ retinal explants with an age-matched wild-type or slit-deficient lens. Numbers above bars indicate numbers of explants analyzed in four independent experiments. ${ }^{*} p<0.05$ compared with wild-type lens. F, G, Quantification of the extent of axon outgrowth in cocultures of retinal explants with mock- or Slit1-transfected cells. Results are the mean of four independent experiments. ${ }^{*} p<0.001$ compared with control. VT, Ventrotemporal; VN, ventronasal; DT, dorsotemporal; DN, dorsonasal.

ance cue expression and function and heparan sulfate proteoglycans (HSPGs) have been found to bind to Slits and play an important role in regulating Slit signaling and axon guidance in vivo (Liang et al., 1999; Hu, 2001; Inatani et al., 2003; Bülow and Hobert, 2004; Johnson et al., 2004; Kantor et al., 2004; Lee et al., 2004; Steigemann et al., 2004). In the future, it will be important to establish whether Slits also interact directly with CSPGs and whether the defects induced by removal of chondroitin sulfates are the result of disrupting Slit localization or function.

\section{Slits regulate distinct aspects of intraretinal guidance within different regions of the retina}

There are many known developmental differences between dorsal and ventral retina. These regions are defined by differential expression of transcription factors, including Tbx5 (T-box 5) and Vax2 (ventral anterior homeobox 5) (Barbieri et al., 1999; Sowden et al., 2001). Retinoid-metabolizing enzymes also are ex- pressed differentially between dorsal and ventral retina ( $\mathrm{Li}$ et al., 2000; Wagner et al. 2000). The eye does not grow symmetrically around the optic disc, and the first RGCs are generated in dorsal retina, followed by differential expansion of the OFL along the dorsoventral axis (Halfter et al., 1985). Many guidance cues also are expressed differentially within dorsal and ventral retina, and this is related to both the topographic mapping of the axons within their targets and regulation of intraretinal pathfinding within specific regions of the retina (Liu et al., 2003; McLaughlin and O'Leary, 2005). Furthermore, several cues that are uniformly expressed throughout the retina are required specifically for guidance within either dorsal or ventral regions (Ott et al., 1998; Birgbauer et al., 2000). After exiting the eye, axons from dorsal and ventral retina are initially segregated but become intermingled at the chiasm before reorganizing and becoming segregated again within the optic tract (Chan and Guillery, 1994; Chan and Chung, 1999). Whether Slits are involved in regulating this aspect of RGC axon guidance has not been established (Thompson et al., 2006). Dorsoventral asymmetries in the distribution of cone classes also occur, suggesting that these regions of the retina serve different visual functions (Szel et al., 1996).

Unexpectedly, we found that Slits are required within both dorsal and ventral retina but mediate distinct aspects of intraretinal guidance. The reason for this regional specificity is not known. Whereas slit1 is expressed in a high ventral-low dorsal gradient, slit2, robo 1 , and robo 2 are distributed uniformly (Erskine et al., 2000). Furthermore, both dorsal and ventral RGCs mediate Slit signaling. The function of individual guidance cues is dependent critically on the context in which they are encountered. The composition of the extracellular environment (Höpker et al., 1999; Nguyen-Ba-Charvet et al., 2001; Kantor et al., 2004) as well as intrinsic factors such as the age of the axons (Shewan et al., 2002) can modulate guidance responses. HSPGs with distinct heparan sulfate structures are important modulators of Slit localization and function ( $\mathrm{Hu}, 2001$; Inatani et al., 2003; Bülow and Hobert, 2004; Johnson et al., 2004; Steigemann et al., 2004), and specific heparin-sulfatemodifying enzymes are expressed in distinct region of the optic pathway (Irie et al., 2002). Thus, one possibility is that the localization of individual heparan sulfate structures may modulate the response of dorsal and ventral RGCs to Slits. Other guidance cues that function redundantly with Slits may also compensate differentially for loss of Slits within dorsal and ventral retina. This suggests that the regional specificity of Slits is likely to be determined by the other factors present in the local environment and highlights the complexity of the mechanisms regulating axon guidance. 


\section{References}

Bagri A, Marin O, Plump AS, Mak J, Pleasure SJ, Rubenstein JL, TessierLavigne M (2002) Slit proteins prevent midline crossing and determine the dorsoventral position of major axonal pathways in the mammalian forebrain. Neuron 33:233-248.

Barbieri AM, Lupo G, Bulfone A, Andreazzoli M, Mariani M, Fougerousse F, Consalez GG, Borsani G, Beckmann JS, Barsacchi G, Ballabio A, Banfi S (1999) A homeobox gene, vax2, controls the patterning of the eye dorsoventral axis. Proc Natl Acad Sci USA 96:10729-10734.

Birgbauer E, Cowan CA, Sretavan DW, Henkemeyer M (2000) Kinase independent function of EphB receptors in retinal axon pathfinding to the optic disc from dorsal but not ventral retina. Development 127:1231-1241.

Brittis PA, Silver J (1995) Multiple factors govern intraretinal axon guidance: a time-lapse study. Mol Cell Neurosci 6:413-432.

Brittis PA, Canning DR, Silver J (1992) Chondroitin sulfate as a regulator of neuronal patterning in the retina. Science 255:733-736.

Brittis PA, Lemmon V, Rutishauser U, Silver J (1995) Unique changes of ganglion cell growth cone behaviour following cell adhesion molecule perturbations: a time-lapse study of the living retina. Mol Cell Neurosci 6:433-439.

Brose K, Bland KS, Wang KH, Arnott D, Henzel W, Goodman CS, TessierLavigne M, Kidd T (1999) Slit proteins bind Robo receptors and have an evolutionarily conserved role in repulsive axon guidance. Cell 96:795-806.

Bülow HE, Hobert O (2004) Different sulfations and epimerization define heparan sulfate specificity in nervous system development. Neuron 41:723-736.

Chan SO, Chung KY (1999) Changes in axons arrangement in the retinofugal pathway of mouse embryos: confocal microscopy study using singleand double-dye label. J Comp Neurol 406:251-262.

Chan SO, Guillery RW (1994) Changes in fiber order in the optic nerve and tract of rat embryos. J Comp Neurol 344:20-32.

Deiner MS, Kennedy TE, Fazeli A, Serafini T, Tessier-Lavigne M, Sretavan DW (1997) Netrin-1 and DCC mediate axon guidance locally at the optic disc: loss of function leads to optic nerve hypoplasia. Neuron 19:575-589.

Erskine L, Williams SE, Brose K, Kidd T, Rachel R, Goodman CS, TessierLavigne M, Mason CA (2000) Retinal ganglion cell axon guidance in the mouse optic chiasm: expression and function of Robos and Slits. J Neurosci 20:4975-4982.

Goldberg S (1977) Unidirectional, bidirectional and random growth of embryonic optic axons. Exp Eye Res 25:399-404.

Halfter W, Deiss S, Schwarz U (1985) The formation of the axonal pattern in the embryonic avian retina. J Comp Neurol 232:466-480.

Holmes GP, Negus K, Burridge L, Raman S, Algar E, Yamada T, Little MH (1998) Distinct but overlapping expression patterns of two vertebrate slit homologs implies functional roles in CNS development and organogenesis. Mech Dev 79:57-72.

Höpker VH, Shewan D, Tessier-Lavigne M, Poo MM, Holt C (1999) Growth cone attraction to netrin-1 is converted to repulsion by laminin-1. Nature 401:69-73.

$\mathrm{Hu} \mathrm{H}$ (2001) Cell-surface heparan sulfate is involved in the repulsive guidance activities of Slit2 protein. Nat Neurosci 4:695-701.

Huminiecki L, Gorn M, Suchting S, Poulsom R, Bicknell R (2002) Magic roundabout is a new member of the roundabout receptor family that is endothelial specific and expressed at sites of active angiogenesis. Genomics 79:547-552.

Inatani M, Irie F, Plump AS, Tessier-Levigne M, Yamaguchi Y (2003) Mammalian brain morphogenesis and midline axon guidance require heparan sulfate. Science 302:1044-1046.

Irie A, Yates EA, Turnbull JE, Holt CE (2002) Specific heparan sulfate structures involved in retinal axon targeting. Development 129:61-70.

Itoh A, Miyabayashi T, Ohno M, Sakano S (1998) Cloning and expressions of three mammalian homologues of Drosophila slit suggest possible roles for Slit in the formation and maintenance of the nervous system. Mol Brain Res 62:175-186.

Jin Z, Zhang J, Klar A, Chédotal A, Rao Y, Cepko CL, Bao ZZ (2003) Irx4mediated regulation of Slit1 expression contributes to the definition of early axonal paths inside the retina. Development 130:1037-1048.

Johnson KG, Ghose A, Epstein E, Lincecum J, O'Connor MB, Van Vactor D (2004) Axonal heparan sulphate proteoglycans regulate the distribution and efficiency of the repellent Slit during midline axon guidance. Curr Biol 14:499-504.

Kantor DB, Chivatakarn O, Peer KL, Oster SF, Inatani M, Hansen MJ, Flanagan JG, Yamaguchi Y, Sretaven DW, Giger RJ, Kolodkin AL (2004) Semaphorin $5 \mathrm{~A}$ is a bifunctional axon guidance cue regulated by heparan and chondroitin sulfate proteoglycans. Neuron 44:961-975.

Kidd T, Brose K, Mitchell KJ, Fetter RD, Tessier-Lavigne M, Goodman CS, Tear G (1998) Roundabout controls axon crossing of the CNS midline and defines a novel subfamily of evolutionarily conserved guidance receptors. Cell 92:205-215.

Kolpak A, Zhang J, Bao ZZ (2005) Sonic Hedgehog has a dual effect on the growth of retinal ganglion axons depending on its concentration. J Neurosci 25:3432-3441.

Lee JS, von der Hardt S, Rusch MA, Stringer SE, Stichney HL, Talbot WS, Geisler R, Nüsslein-Volhardt C, Selleck SB, Chien CB, Roehl H (2004) Axon sorting in the optic tract requires HSPG synthesis by ext2 (dackel) and extl3 (boxer). Neuron 44:947-960.

Li H, Wagner E, McCaffery P, Smith D, Andreadis A, Drager UC (2000) A retinoic acid synthesizing enzyme in ventral retina and telencephalon of the embryonic mouse. Mech Dev 95:283-289.

Li HS, Chen JH, Wu W, Fagaly T, Zhou L, Yuan W, Dupuis S, Jiang ZH, Nash W, Gick C, Ornitz DM, Wu JY, Rao Y (1999) Vertebrate Slit, a secreted ligand for the transmembrane protein Roundabout, is a repellent for olfactory bulb axons. Cell 96:807-818.

Liang Y, Annan RS, Carr SA, Popp S, Mevissen M, Margolis RK, Margolis RU (1999) Mammalian homologues of the Drosophila slit protein are ligands of the heparan sulfate proteoglycan glypican-1 in brain. J Biol Chem 274:17885-17892.

Liu J, Wilson S, Reh T (2003) BMP receptor $1 \mathrm{~b}$ is required for axon guidance and cell survival in the developing retina. Dev Biol 256:34-47.

Long H, Sabatier C, Ma L, Plump A, Yuan W, Ornitz DM, Tamada A, Murakami F, Goodman CS, Tessier-Lavigne M (2004) Conserved roles for Slit and Robo proteins in midline commissural axon guidance. Neuron 42:213-223.

Marcus RC, Blazeski R, Godemont P, Mason CA (1995) Retinal axon divergence in the optic chiasm: uncrossed axons diverge from crossed axons within a midline glial specialization. J Neurosci 15:3716-3729.

McAdams BD, McLoon SC (1995) Expression of chondroitin sulfate and keratan sulfate proteoglycans in the path of growing retinal axons in the developing chick. J Comp Neurol 352:594-606.

McLaughlin T, O’Leary DD (2005) Molecular gradients and development of retinotopic maps. Annu Rev Neurosci 28:327-355.

Monnier PP, Beck SGM, Bolz J, Henke-Fahle S (2001) The polysialic acid moiety of the neural cell adhesion molecule is involved in the intraretinal guidance of retinal ganglion cell axons. Dev Biol 229:1-14.

Nguyen-Ba-Charvet KT, Brose K, Marillat V, Kidd T, Goodman CS, Tessier-Lavigne M, Sotelo C, Chédotal A (1999) Slit2-mediated chemorepulsion and collapse of developing forebrain axons. Neuron 22:463-473.

Nguyen-Ba-Charvet KT, Brose K, Marillat V, Sotelo C, Tessier-Lavigne M, Chédotal A (2001) Sensory axon response to substrate-bound Slit2 is modulated by laminin and cyclic GMP. Mol Cell Neurosci 17:1048-1058.

Nguyen-Ba-Charvet KT, Plump AS, Tessier-Lavigne M, Chédotal A (2002) Slit1 and Slit2 proteins control the development of the lateral olfactory tract. J Neurosci 22:5473-5480.

Niclou SP, Jia L, Raper JA (2000) Slit2 is a repellent for retinal ganglion cell axons. J Neurosci 20:4962-4974.

Ohta K, Tannahill D, Yoshida K, Johnson AR, Cook GMW, Keynes RJ (1999) Embryonic lens repels retinal ganglion cell axons. Dev Biol 211:124-132.

Ott H, Bastmeyer M, Stuermer CAO (1998) Neurolin, the goldfish homolog of DM-GRASP, is involved in retinal axon pathfinding to the optic disk. J Neurosci 18:3363-3372.

Plump AS, Erskine L, Sabatier C, Brose K, Epstein CJ, Goodman CS, Mason CA, Tessier-Lavigne M (2002) Slit1 and Slit2 cooperate to prevent premature midline crossing of retinal axons in the mouse visual system. Neuron 33:219-232.

Ring C, Lemmon V, Halfter W (1995) Two chondroitin sulfate proteoglycans differentially expressed in the developing chick visual system. Dev Biol 168:11-27.

Ringstedt T, Braisted JE, Brose K, Kidd T, Goodman C, Tessier-Lavigne M, O'Leary DDM (2000) Slit inhibition of retinal axon growth and its role 
in retinal axon pathfinding and innervation patterns in the diencephalon. J Neurosci 20:4983-4991.

Sabatier C, Plump AS, Ma L, Brose K, Tamada A, Murakami F, Lee EY, Tessier-Lavigne M (2004) The divergent Robo family protein Rig1/ Robo3 is a negative regulator of slit responsiveness required for midline crossing by commissural axons. Cell 117:157-169.

Shewan D, Dwivedy A, Anderson R, Holt CE (2002) Age-related changes underlie switch in netrin-1 responsiveness as growth cones advance along visual pathway. Nat Neurosci 5:955-962.

Sowden JC, Holt JK, Meins M, Smith HK, Bhattacharya SS (2001) Expression of Drosophila omb-related T-box genes in the developing human and mouse neural retina. Invest Ophthalmol Vis Sci 42:3095-3102.

Steigemann P, Molitor A, Fellert S, Jäckle H, Vorbrüggen G (2004) Heparan sulfate proteoglycan syndecan promotes axonal and myotube guidance by Slit/Robo signalling. Curr Biol 14:225-230.

Stier H, Schlosshauer B (1995) Axonal guidance in the chicken retina. Development 121:1443-1454.

Stier H, Schlosshauer B (1998) Different cell surface areas of polarized radial glia having opposite effects on axonal outgrowth. Eur J Neurosci 10:1000-1010.
Szel A, Rohlich P, Caffe AR, van Veen T (1996) Distribution of cone photoreceptors in the mammalian retina. Microsc Res Tech 35:445-462.

Thompson H, Barker D, Camand O, Erskine L (2006) Slits contribute to the guidance of retinal ganglion cell axons in the mammalian optic tract. Dev Biol, in press.

Wagner E, McCaffery P, Dräger UC (2000) Retinoic acid in the formation of the dorsoventral retina and its central projections. Dev Biol 222:460-470.

Yuan SSF, Cox LA, Dasika GK, Lee EYHP (1999a) Cloning and functional studies of a novel gene aberrantly expressed in RB-deficient embryos. Dev Biol 20:62-75.

Yuan W, Zhou L, Chen JH, Wu JY, Rao Y, Ornitz DM (1999b) The mouse SLIT family: secreted ligands for ROBO expressed in patterns that suggest a role in morphogenesis and axon guidance. Dev Biol 212:290-306.

Zhang J, Jin Z, Bao ZZ (2004) Disruption of gradient expression of Zic3 resulted in abnormal intraretinal axon projection. Development 131:1553-1562.

Zelina P, Hasan XA, Karsten T, Pollerberg E (2005) The cell adhesion molecule NrCAM is crucial for growth cone behaviour and pathfinding of retinal ganglion cell axons. Development 132:3609-3618. 